\title{
Prácticas en la enseñanza de la escritura argumentativa académica. La estructura textual
}

\author{
Vera J. Moreno-Fontalvo \\ Facultad de Ciencias Humanas y Sociales, Departamento de Humanidades, Universidad de la Costa, Calle 58 \# 55-66, \\ Barranquilla, Colombia. (correo-e: vmoreno4@cuc.edu.co)
}

Recibido Jul. 11, 2019; Aceptado Sep. 4, 2019; Versión final Nov. 5, 2019, Publicado Abr. 2020

\begin{abstract}
Resumen
El propósito de esta investigación es analizar las prácticas de escritura académica en estudiantes que cursan la asignatura de construcción textual de la Universidad de la Costa en la ciudad de Barranquilla, Colombia. La muestra se constituyó de 9 estudiantes, correspondientes al grupo finalista de la Tercera versión de las Olimpiadas Escriturales. Estas corresponden a un proyecto en el que los participantes construyen un texto argumentativo, guiado por mentores, de los cuales reciben retroalimentación para la mejora de su escrito. Este estudio abordó un enfoque mixto donde se articula un componente cualitativo a través de la técnica análisis documental con un componente cuantitativo, mediante el análisis estadístico descriptivo. Para el análisis de los datos se utilizó el software Statistical Product and Service Solutions (SPSS). Los resultados confirman que el uso de una estructura textual tipo argumentativa permite mayor dominio en la composición escrita. Se concluyó que tener claridad sobre las etapas que conforman la estructura textual argumentativa impacta positivamente en las producciones textuales de los estudiantes.
\end{abstract}

Palabras clave: escritura académica; texto argumentativo; estructura textual; género discursivo; alfabetización académica

\section{Practices in the teaching of academic argumentative writing. The textual structure}

\begin{abstract}
The purpose of this research is to analyze the practices of academic writing in students taking the subject of textual construction of the University of the Coast in the city of Barranquilla, Colombia. The sample consisted of 9 students, corresponding to the finalist group of the third version of the Scriptural Olympics; These are a project in which the participants construct an argumentative text, guided by mentors and, from which they receive feedback for the improvement of their writing. This study addressed a mixed approach where a qualitative component is articulated through the technique documentary analysis with a quantitative component, through descriptive statistical analysis. The Statistical Product and Service Solutions software (SPSS) was used to analyze the data. The results confirm that the use of an argumentative textual structure allows greater mastery in the written composition. It was concluded that having clarity about the stages that make up the argumentative textual structure positively impacts students' textual productions.
\end{abstract}

Keywords: academic writing; argumentative text; textual structure; discursive genre; academic literacy 


\section{INTRODUCCIÓN}

Escribir es una actividad muy frecuente en el ámbito universitario. Gran parte del conocimiento, se elabora a través de textos leídos en las diferentes disciplinas. El desarrollo de un discurso académico, para cualquier estudiante, se convierte en el instrumento de construcción del conocimiento y de las relaciones que establece con las interacciones de su entorno. En palabras de Halliday (1993), el discurso es el elemento central del conocimiento, que a través de las experiencias sobreviene el conocimiento. En la construcción de conocimiento, una misma persona participa en varias actividades que pueden involucrar diferentes ámbitos. En una actividad social, por ejemplo, los géneros discursivos son herramientas de participación. Dentro de estos ejercicios, se aprende a usar la lengua en circunstancias determinadas que requieren de habilidades lingüístico-comunicativas. En el aprendizaje de la lengua es indispensable aprender a usarla de forma adecuada en cada una de las interacciones que el individuo ejecuta con su medio. Durante esa participación social, el ejercicio de la escritura implica tener clara una situación de aprendizaje que conlleve la activación de saberes a través de la conexión con la realidad circundante. Al involucrarse en una situación contextualizada, el individuo analiza e interpreta su propia realidad, provocando en él, reflexiones que le obligan a tomar decisiones sobre posibles alternativas de solución en la problemática analizada, volviéndose más creativo en cada proposición (Lave y Wenger,1991).

Puede decirse entonces, que el aprendizaje situado en el contexto educativo exige una forma diferente de desarrollarlo. En el ámbito universitario, por ejemplo, se le debe ofrecer al educando experiencias significativas y reales; de tal forma que los sujetos interactúen bajo condiciones especiales y guiados mediante un proceso de andamiaje que facilite su desempeño dentro de la experiencia a la que ha estado expuesto. Por ello, es importante reconocer el valor de un aprendizaje situado como eje fundamental para la construcción textual, porque el atender una problemática como tópico serán capaces de aplicar de forma consciente y según la intención comunicativa, las reglas lingüísticas que caracterizan el tipo de escrito. En la educación superior, los estudiantes crean textos con características propias a un determinado género discursivo, conforme a ciertos saberes específicos y atendiendo a intenciones particulares que los hacen funcionales, lo cual implica saber hacerlo (Camps y Montserrat, 2013). Por eso es importante que el estudiante conozca las características propias de los géneros discursivos porque en la práctica se convierten en el insumo más utilizado al enfrentarse a la escritura de un texto académico (Rayas y Méndez, 2017).

El propósito de la escritura en el contexto universitario está relacionado con la función educativa que cumple toda universidad, desde lo formativo y/o lo investigativo, interrelacionándose frecuentemente. Dichas funciones determinan las prácticas discursivas escritas en las que participan los estudiantes y, con seguridad también, los profesores. Estas dos funciones son a su vez dos grandes grupos de actividades de las que se desligan subgrupos relacionados con las diversas disciplinas y ámbitos científicos y profesionales implicados. Este trabajo responde a las funciones mencionadas, pues es producto del análisis y la reflexión sobre cómo escriben los estudiantes en educación superior. Se piensa que producir e interpretar textos escritos es un tema concluido al ingresar a la universidad, sin reconocer que en este ámbito se trabajan diversas temáticas y tipos de textos, los cuales reflejan intenciones y propósitos variados en contextos determinados lo que lleva a crear nuevos desafíos y exigencias al estudiante. Se suma a ello, que, en ocasiones, se observen ciertas contradicciones generadas por interrelacionar docencia con investigación, además de las que se derivan de percepciones disciplinares muy particulares sobre las prácticas de escritura.

Algunas personas aluden que la enseñanza de la escritura es responsabilidad del docente de lengua castellana, he aquí el ejemplo de una de las contradicciones, ya que no es posible pensar de forma unilateral que solo un área sea capaz de trabajar cuando sería importante analizar que todas las áreas del saber deben involucrarse en el proceso de construcción. Son los especialistas de la disciplina los que a través de su conocimiento disciplinar pueden apoyar el aprendizaje de lectura y escritura. La retroalimentación del experto contribuye a la autorregulación del estudiante, permitiendo que por cada comentario se mejore la presentación del escrito posterior (Padilla y López, 2019). La autorregulación ayuda al educando a desarrollar competencias. Ser competente a nivel escritural implica tener presente los contextos, los pre-saberes y saberes sobre el tema, el tipo de texto, al igual que la estructuración y redacción de las ideas en contextos auténticos que viabilicen la aplicación de estrategias afectivas, de cognición y metacognición con las que el estudiante logre enfrentar situaciones durante la producción textual (Pastene et al., 2016 y López et al., 2016).

Aprender a leer y escribir en el ámbito académico y en especial en educación superior, exige profundidad del saber, autonomía y consulta bibliográfica, lo que implica aplicar procesos mentales para comprender, interiorizar información y desarrollar pensamiento. Desde perspectivas actuales, escribir requiere de conocer el código escrito, las normas establecidas que dan cuenta de ortografía, sintaxis, coherencia y cohesión, además del empleo de habilidades cognitivas y la interacción sociocultural. El buen manejo de los aspectos descritos contribuye, también en el proceso de comprensión de quien escribe (Peña, 2011). 
Desarrollar procesos de escritura no es tan fácil, no se trata de enfatizar en asuntos gramaticales, el proceso requiere de enlazarlos con otros elementos. Es necesario reconocer que los procesos de lectura y escritura deben permanecer como prácticas diarias para la construcción del conocimiento dentro y fuera el aula de clase, apropiarse de información extra invita a la reelaboración de lo escrito, a la interpretación y análisis de textos académicos que enriquezcan las ideas primarias. Autores como Carlino (2013) y Torres (2018), piensan que la escritura es una actividad que se produce en condiciones sociales desde diferentes disciplinas, así la enseñanza de esta debe ser compartida.

Entre las variadas formas de escritura académica, en este estudio se ha enfatizado lo concerniente a la argumentación. Al respecto, la acción argumentativa en el ámbito académico, tiene correspondencia con ciertos recursos discursivos que deben evidenciar procesos cognitivos como la observación, interpretación, comparación, descripción, análisis y síntesis, entre otros. Acerca de la secuencia argumentativa, ésta, ha sido abordada por varios autores, para esta investigación, se utilizó la estructura textual trabajada por Van Dijk (1992), Weston (2006), Errázuriz (2014), Díaz (2014) y Ruiz (2014), con una estructura argumentativa genérica que se ha representado en un esquema (Figura 1), que incluyera las diferentes percepciones de los autores mencionados.

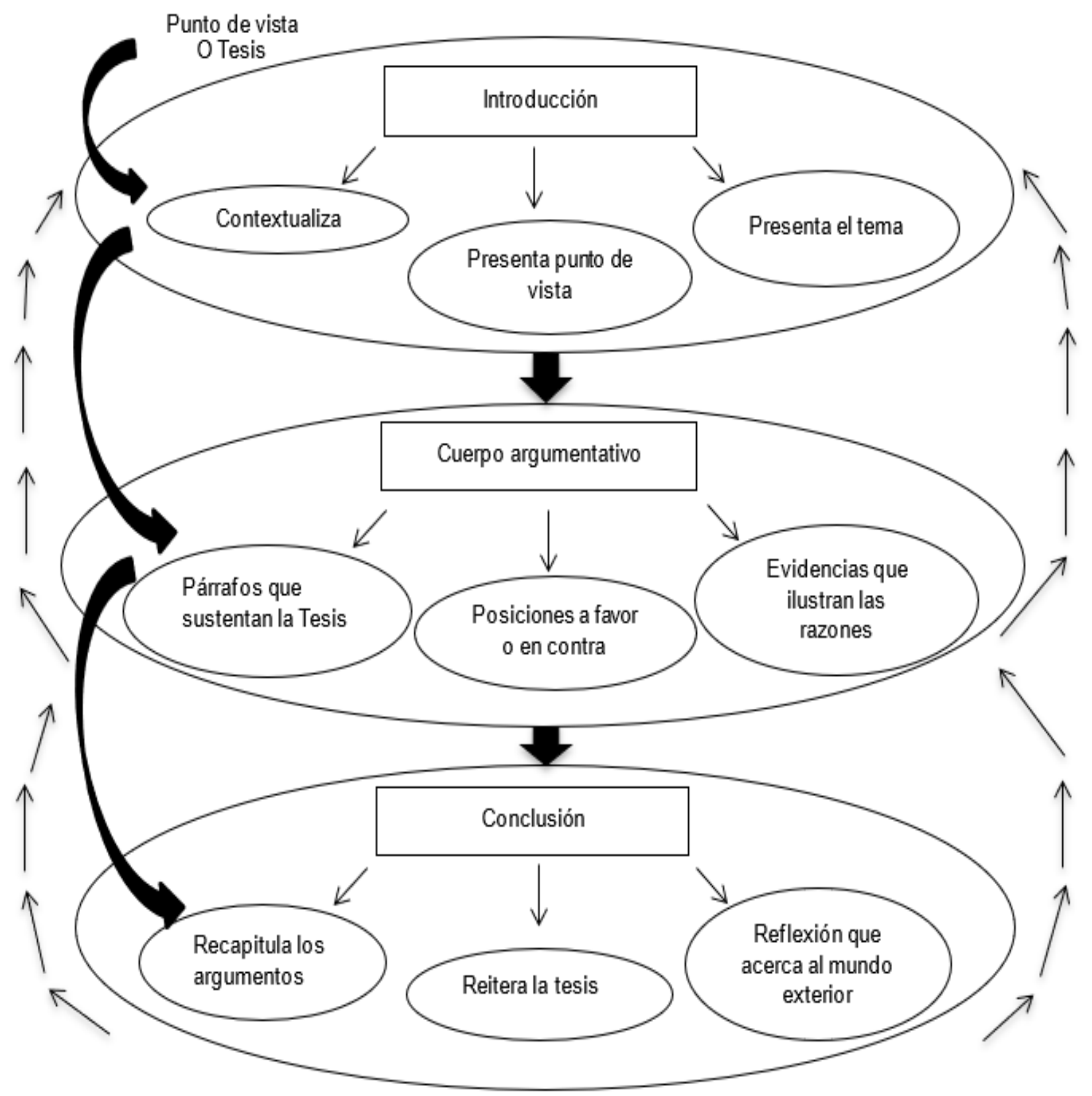

Fig. 1: Estructura argumentativa genérica del equipo experimental usado 
Desde esas perspectivas, con el propósito de direccionar la enseñanza-aprendizaje hacia una pedagogía del texto, se determina que los elementos contenidos de la estructura textual son: introducción, cuerpo argumentativo y conclusión.

Introducción: presenta el tema y contextualiza, estableciendo antecedentes o generalidades del tópico para despertar el interés del lector sobre lo que se leerá en el desarrollo del texto. Deja ver el tono en que el escritor aborda el tema, el cual está ligado al propósito del texto: persuadir. Aunque no se considera obligación, una vez terminada la introducción se presenta la tesis; no obstante, ésta, también puede encontrarse en el desarrollo o al final del texto, -para este estudio se abordó un tesis explícita-. La tesis, es un juicio de valor que se redacta con el objeto de asumir una posición frente al tópico; es decir, punto de vista. Debe escribirse con total claridad y precisión. Para Díaz, (2014) la tesis es una proposición expresa o implícita que representa el punto de vista del autor de un tema particular.

Cuerpo argumentativo: este aparte del texto lo integran los diferentes párrafos que justifican la tesis planteada, las razones que se exponen para sustentarla son los argumentos; éstos, muestran las posiciones a favor y/o en contra. Es importante presentar argumentos que se desarrollen en párrafos independientes, sin embargo, en algunas ocasiones un argumento, podría escribirse en dos o tal vez tres párrafos continuos para evitar saturarlos de información y que resulten muy extensos. La sustentación del punto de vista es prioridad en el proceso de argumentación, por tal razón, se espera que todo autor favorezca al lector la comprensión de su texto, en este caso que logre entender la tesis; para ello, debe ilustrar las razones que expone empleando evidencias (datos estadísticos, hechos, ejemplos, analogías, citas de autoridad) como soporte a su tesis.

Conclusión: recapitula los argumentos y por ende reitera la tesis. Puede establecerse como una reflexión sobre el tópico, una sugerencia o recomendación para afrontar la problemática abordada; esto es, el "Deber ser", una idea o ideas que conecten al autor con el mundo exterior. Por otro lado, es importante mencionar que en el proceso de argumentación subyacen términos como texto y discurso, así, el primero hace referencia a los recursos formales de la cohesión y a los aspectos de la sintaxis para conectar I información; en cambio, el segundo corresponde a lo pragmático de la lengua, es decir a la coherencia. Por lo tanto, argumentar requiere del uso consciente de estrategias lingüísticas (Ruiz, 2014). Sobre la cohesión, Barletta y Chamorro (2010) refieren acerca de la presencia de los vínculos y las transiciones como elementos cohesivos, señalando que en un texto suelen observarse a manera de párrafos, ideas sencillas que cumplen la función de establecer conexión con lo dicho anteriormente o advertir lo que prosigue en el escrito.

En otro orden de ideas, los aspectos más relevantes en el desarrollo de prácticas referidas a la escritura, y haciendo énfasis en el tipo de texto escogido, estarían dadas bajo tres elementos: instrucción, tiempo y colaboración. La Instrucción, comprende cualquier tipo de orientación brindada a los estudiantes. El tiempo, corresponde a todos los acuerdos relativos a los plazos de escritura. La colaboración indica todas las relaciones y las interacciones que surgen de la escritura (kruse, 2013). Estos tiempos pueden presentarse en cualquier nivel de educación. A propósito del nivel superior, cuando un estudiante ingresa a la universidad debe aprender a convivir con las exigencias de ésta, sabe que será necesario enfrentarse a cambios; la apropiación de gran cantidad de información, por ejemplo, desde las distintas disciplinas de estudio puede convertirse en un obstáculo si no se tienen las condiciones adecuadas para asumirlas. En especial, si se trata de desarrollar argumentación. La argumentación disciplinar conlleva a la exploración, elaboración e información de conocimientos efectuados por los que integran agrupaciones científicas, mediante el empleo del discurso académico (Hyland, 2012).

Dentro de cada área disciplinar, los jóvenes tendrían que seguir un modelo de alfabetización académica que involucre el uso de discursos específicos, ya sea escrito u oral, es decir, hacer alusión a la enseñanza de la lectura y la escritura en una determinada cultura letrada desde una comunidad académica en particular. Castells et al. (2015) y Pereira (2018) indican que, en el caso de la escritura académica, un estudiante debe tener claridad sobre cómo se hace y asumir el reto de escribir de forma auténtica, reconociéndose como actor competente para que pueda producir textos con altos niveles de calidad. En suma, la variedad de herramientas requeridas para interactuar en la cultura discursiva de las áreas del saber, así como cualquier actividad de interpretación y construcción textual necesarias para el aprendizaje en la universidad hace referencia a la alfabetización académica, y éste es un proceso que debe ser guiado por el docente para cerrar las brechas existentes entre cómo leen y cómo escriben los estudiantes de primer ingreso a la universidad (Carlino,2003).

Lo planteado llevó a formular la pregunta: ¿De qué manera las prácticas de escritura efectuadas por los estudiantes evidencian sus competencias sobre la estructura textual argumentativa?, pues busca establecer revisión de las prácticas escriturales en el caso específico, el texto argumentativo. 


\section{METODOLOGÍA}

El trabajo aborda un enfoque Mixto. La técnica empleada de orden cualitativo fue el análisis documental y en cuanto al orden cuantitativo se trabajó con estadística descriptiva. El análisis estadístico de los datos recopilados se efectuó mediante el cálculo de la media y la desviación estándar para observar la fluctuación de los datos respecto a su punto central o media; en específico, para examinar el promedio de diferencia existente entre los diferentes criterios de las variables objeto de estudio y la desviación estándar que establece sobre el grado de dispersión de los datos con respecto a la media. Para establecer la correlación de los datos se utilizó el software Startical Product and Service Solutions, SPSS. Mediante el programa SPSS se determina que el número decimal obtenido al vincular las variables, evidencia el grado de relación y significancia estadística de éstas. La desviación típica será baja si los valores se acercan a cero; indicando esto que los resultados son muy parecidos, será alta, si los valores se alejan de cero; por lo tanto, existirá una gran variación y el grado de exactitud será menor.

Es importante señalar que la investigación surge por el desarrollo de un proyecto titulado "Olimpiadas Escriturales", aplicado en la Universidad de la Costa de la ciudad de Barranquilla, Colombia. Los estudiantes de la asignatura de Construcción Textual, en esta tercera versión de las Olimpiadas Escriturales fueron sometidos a una prueba diagnóstica, revisada por los 4 profesores de la asignatura en mención, distribuidos en los 9 cursos existentes; éstos, se encargaron de seleccionar los trabajos con apropiadas condiciones de escritura como planificación, organización (estructura textual), postura sobre el tema y progresión temática. El grupo de jóvenes seleccionados -146- se subdividieron en 9 grupos y asistieron a unas horas de trabajo con un profesor que fungió como mentor. Desde la perspectiva del proyecto, la mentoría reunía al novato (estudiante) con el experto (profesor), quien estimulaba y ofrecía apoyo en aras de desarrollar competencias escriturales en el mentorado.

Para esta versión de las Olimpiadas Escriturales se contrataron 9 profesores -mentores- del área de lengua castellana. Asistieron a dos sesiones de inducción con el propósito de formarlos en el uso de la rúbrica y explicarles las generalidades del proyecto. En esta ocasión, 4 de los 9 mentores habían participado en la segunda versión de las Olimpiadas Escriturales, por lo tanto, conocían la rúbrica. Se trabajaron Una vez se presentaron los encuentros con los estudiantes, el mentor brindó las orientaciones respectivas para la producción del texto argumentativo. El tema central del texto que debía construirse estuvo relacionado con la línea de investigación Institucional: Desarrollo Sostenible. Así que, se escogió el objetivo 11 de Desarrollo Sostenible para efectuar las consultas pertinentes y elaborar el texto.

Cabe anotar, que el propósito de las Olimpiadas Escriturales era que los participantes iniciaran una competición para la construcción del texto. Se trabajaron 30 sesiones, 20 presenciales y 10 virtuales. Las jornadas se desarrollaban por etapas, durante las cuales, se iban eliminando los estudiantes que no adecuaran su escrito según las normas establecidas por la rúbrica de evaluación. En el proceso de eliminación los mentores revisaban los avances del texto, teniendo en cuenta las etapas redactadas, acorde a los criterios de valoración del instrumento empleado para la revisión de los textos: la rúbrica. Al finalizar la última etapa del proyecto, el mentor trabajó con el mejor de su grupo, es decir, el escrito finalista.

Este escrito fue enviado a tres jurados expertos, quienes en su currículo evidenciaban una alta trayectoria en la revisión de textos académicos. Ellos evaluaron e indicaron los ganadores de las "Olimpiadas Escriturales". Los textos ganadores fueron utilizados para el análisis del estudio, a este respecto, la metodología propende efectuar una descripción de los textos escritos; más específico, las etapas del texto argumentativo. En ese orden de ideas, puede afirmarse que la muestra de esta investigación fue intencional, constituida por los 9 estudiantes, correspondientes al grupo finalista -ganadores de las Olimpiadas Escriturales-

Retomando el instrumento de evaluación, la rúbrica fue sometida a juicio de experto (tres lingüistas). El diseño de la rúbrica tenía el propósito de evaluar las etapas de la estructura del texto argumentativo. Se le asignó un valor a cada etapa; se efectuó una prueba piloto para determinar su confiabilidad. Siendo validada se empleó en las clases de Construcción Textual y en las primeras dos versiones de las Olimpiadas Escriturales. EI estudio de las prácticas de escritura se realizó teniendo en cuenta aspectos como: título, introducción, tesis, argumentos, conclusión, gramática-redacción y fuentes bibliográficas (tabla 1). La rúbrica puede convertirse en una herramienta efectiva para la revisión y control del trabajo de los estudiantes, desde el punto de vista formativo; como también en una matriz de valoración referida a la evaluación (Raposo y Martínez, 2011). Para Muñoz y Valenzuela (2011), la presentación de la rúbrica hace correspondencia a una lista de descriptores que mide tanto elementos de organización como de estructura textual. 
Tabla 1: Rúbrica para evaluar texto argumentativo académico del equipo experimental usado

\begin{tabular}{|c|c|c|}
\hline Etapas & Criterios de valoración & Valoración \\
\hline Título & Es llamativo y está relacionado con el tema tratado. & 0.5 \\
\hline Introducción & $\begin{array}{l}\text { Plantea el tema. } \\
\text { Presenta información muy pertinente y contextualizada según el tema abordado. } \\
\text { Logra llamar la atención con algún recurso discursivo: afirmación fuerte, una cita } \\
\text { relevante, una estadística o una pregunta dirigida al lector. }\end{array}$ & 0.5 \\
\hline Tesis & $\begin{array}{l}\text { Se identifica con claridad. Está en la introducción o inmediatamente después de ésta, } \\
\text { y es una sola. }\end{array}$ & 1.0 \\
\hline Argumentos & $\begin{array}{l}\text { Los argumentos son pertinentes para la tesis que defiende y se plantean con claridad. } \\
\text { Las ideas presentadas son propias, sustentadas con evidencias (hecho, estadísticas, } \\
\text { ejemplos, citas, experiencia de la vida real) que apoyan la tesis. } \\
\text { Al menos dos argumentos se fundamentan con fuentes de carácter bibliográfico. } \\
\text { Los argumentos se desarrollan en párrafos independientes sin perder coherencia ni } \\
\text { cohesión. } \\
\text { Los argumentos y evidencias presentan un orden lógico y están apoyados en } \\
\text { elementos de conexión explícitos y apropiados. }\end{array}$ & 1.5 \\
\hline Conclusión & $\begin{array}{l}\text { La conclusión es fuerte y deja al lector con una idea clara de la posición del autor. } \\
\text { La conclusión reitera la tesis. }\end{array}$ & 0.7 \\
\hline $\begin{array}{l}\text { Gramática } \\
\text { Ortografía, } \\
\text { Puntuación, Léxico } \\
\text { Y Registro. }\end{array}$ & $\begin{array}{l}\text { El texto tiene una adecuada gramática, ortografía, puntuación y edición. } \\
\text { El texto tiene un registro académico. } \\
\text { Presenta variedad de transiciones bien empleadas. }\end{array}$ & 0.5 \\
\hline $\begin{array}{l}\text { Fuentes De } \\
\text { Información }\end{array}$ & $\begin{array}{l}\text { Todas las fuentes usadas son creíbles y están citadas correctamente de acuerdo con } \\
\text { las normas APA. }\end{array}$ & 0.3 \\
\hline
\end{tabular}

\section{RESULTADOS Y DISCUSIÓN}

Desde la estructura del texto y teniendo en cuenta los criterios en la rúbrica de evaluación, el criterio "título" con valoración comprendida entre 0 y 0.5 , tiene una media de 0,4185; una desviación estándar de 0,7658 y un error estándar de 0,02553 con nota máxima de 0,50 y mínima de 0,27. Los valores obtenidos señalan que los títulos redactados fueron pertinentes de acuerdo al tema tratado, presentaban palabras atractivas para el lector que daban sentido a la argumentación. El criterio "introducción" con valoración comprendida entre 0 y 0.5 , tiene una media de 0,4444; una desviación estándar de 0,04082 y un error estándar de 0,01361 con nota máxima de 0,50 y mínima de 0,37. Para Errázuriz (2014) este aparte del texto se muestra al inicio, conformado por elementos que pueden variar según el estilo del autor. Los valores encontrados en el análisis, señalan que uno de los trabajos falló en la contextualización, la información registrada en la introducción fue insuficiente lo que indica que no se ambientó de forma eficiente sobre lo que se leería en el desarrollo del texto.

El criterio "tesis" con valoración comprendida entre 0 y 1 , tiene una media de 0,7630 ; una desviación estándar de 0,17276 y un error estándar de 0,05759 con nota máxima de 0,93 y mínima de 0,47. Este criterio, en 7 de los escritos evaluados fue formulado con claridad y precisión; anunciaba el tema y expresaba un juicio de valor. Todas las tesis fueron explicitas y se anotaron al finalizar de la introducción dejando entrever la actitud o el tono que predominaría en el texto. El criterio "argumentos" con valoración comprendida entre 0 y 1.5 , tiene una media de 1,2111; una desviación estándar de 0,18409 y un error estándar de 0,06136 con nota máxima de 1,43 y mínima de 0,97. El proceso de argumentación suele ser el más difícil en la escritura académica, pues requiere que el estudiante sustente su punto de vista mediante argumentos pertinentes y los desarrolle con coherencia, permitiéndoles mantener progresión temática.

Los resultados obtenidos mostraron textos con adecuada composición argumentativa. La estructura de información y los recursos discursivos coadyuvan a una verdadera dinámica textual, donde, además de lo mencionado, interviene la conexión textual, esta obedece al orden lógico de los componentes semánticopragmáticos empleados y la acción misma argumentativa que ejerce el poder de saber ubicar en el escrito procesos discursivos que dan cuenta de actitudes cognitivas como el análisis, la observación, la composición, la descripción, entre otras (Páez, 2013). De otra parte, partiendo de la perspectiva de Errázuriz (2014) y desde lo planteado por Villarroel, et al. (2019), la secuencia argumentativa sugiere posiciones que pueden estar a favor o en contra de un planteamiento en particular; considerándose de mayor valor estos últimos, ya que hacen parte de la crítica o posición personal con respecto al diálogo entre los autores. Lo anterior indica que la comprensión y el análisis crítico de los planteamientos argumentativos de otros autores, incidió en la construcción de argumentos propios, debidamente sustentados; presentando evidencias como citas de autoridad, datos estadísticos, ejemplos y/o analogías que validaban las aportaciones personales. 
El criterio "conclusión" con valoración comprendida entre 0 y 0.7 , tiene una media de 0,5370 ; una desviación estándar de 0,09044 y un error estándar de 0,03015 con nota máxima de 0,67 y mínima de 0,43. Díaz (2014) indica que los finales buenos le brindan al texto solidez y firmeza. Esta etapa de la estructura permite revisar la fundamentación de la posición de quien escribe y requiere que se lea la interconexión de las ideas planteadas (Ramírez y Zamora, 2014). En los trabajos evaluados, este criterio reflejó gran varianza en los puntajes obtenidos. Las ideas concluyentes que surgieron del desarrollo de los argumentos y daban soporte a la tesis, no fueron contundentes en la mayoría de los textos. Aunque se reiteraba el punto de vista principal, es decir, la tesis, las conclusiones develaban una pobre reflexión sobre lo sustentado faltando carácter y proposición.

El criterio "gramática, ortografía, puntuación, léxico y registro" con valoración comprendida entre 0 y 0.5 , tiene una media de 0,3926; una desviación estándar de 0,06186 y un error estándar de 0,02062 con nota máxima de 0,53 y mínima de 0,33. En cuanto a este criterio hubo una marcada desviación. Los textos indicaban un registro académico, debido al tópico trabajado, el lenguaje fue formal y la terminología de carácter científico, no obstante, en algunos textos se observó falla en el uso de conectores o frases de transición, impidiendo la cohesión de ciertas ideas entre párrafo y párrafo.

El criterio "fuentes" con valoración comprendida entre 0 y 0.3 , tiene una media de 0,2259; una desviación estándar de 0,05212 y un error estándar de 0,01737 con nota máxima de 0,30 y mínima de 0,17. Desde lo establecido en la rúbrica en relación con este criterio, se esperaba que los estudiantes hicieran buen manejo de las fuentes bibliográficas, esto es, citar y referenciar de manera precisa; sin embargo, los valores arrojados en el análisis, muestran una varianza, es decir, 6 de los trabajos obtuvieron bajos resultados, lo que significa que se les dificulta citar correctamente, e incluso muchos alumnos no utilizan las normas de la APA porque desconocen su aplicabilidad al citar o referenciar.

El puntaje "promedio final" de los textos evaluados con valoración comprendida entre 0 y 5.0 , tuvieron una media de 3,9944; una desviación estándar de 0,56723 y un error estándar de 0,18908 con nota máxima de 4,70 y mínima de 3,17. Estos valores indican que los mejores puntajes fueron dados a los textos 5, 4, 8, respectivamente lo que significa que lograron crear un buen discurso escrito y bajo la estructura requerida en los textos argumentativos. Pastene et al. (2016), afirman que todo acto de escritura lleva consigo procesos cognitivos que se acompañan de elementos textuales, dados en contextos determinados y quien escribe requiere de la planificación, organización y edición constante de lo redactado con el fin de alcanzar un trabajo preciso y veraz para quien le corresponda leerlo.

Desde lo anunciado, el estudiante fue capaz de crear textos con un discurso dialógico que involucró factores intersubjetivos e intertextuales que dieron lugar a pensamientos propios desde el razonamiento de puntos de vista convergentes y divergentes haciendo uso de elementos metalingüísticos. Para finalizar por medio de las desviaciones, se pudo observar que de los aspectos revisados en la construcción del texto el que menor desviación tuvo fue la introducción y en su orden le siguen las fuentes, gramática, título, conclusión, tesis y argumentos; lo que quiere decir que en la redacción de argumentos, se tuvieron en cuenta los criterios establecidos, tales como: claridad y pertinencia, según la tesis planteada; presentación de ideas propias, sustentadas con evidencias que apoyaban la tesis respectiva y desarrollo de argumentos en párrafos independientes, sin perder coherencia y cohesión.

\section{DISCUSIÓN FINAL}

De acuerdo con los resultados sistematizados en este estudio, se constató lo siguiente: 1) según la revisión de los trabajos finales en la tercera versión de las Olimpiadas escriturales, se observó que los estudiantes muestran niveles de competencia del proceso de escritura en sus aspectos formales relacionados con el discurso académico del texto argumentativo construido; 2) se identificaron aspectos formales de escritura. Así: Los argumentos fueron pertinentes para la tesis planteada y ésta fue clara. Las ideas presentadas fueron propias, sustentadas con evidencias (hecho, estadísticas, ejemplos, citas, experiencia de la vida real) que apoyaban la tesis. Los argumentos se fundamentaron con fuentes de carácter bibliográfico. Los argumentos se desarrollaron en párrafos independientes sin perder coherencia ni cohesión. Los argumentos y evidencias presentaron un orden lógico y estuvieron apoyados en elementos de conexión explícitos y apropiados; 3) lo que corresponde a las actividades diseñadas para el aprestamiento de la estructura textual, éstas fueron didácticas y permitieron dinamizar el proceso de revisión. En relación a esto último, la retroalimentación se constituyó en una estrategia para mejorar la escritura; en la mayoría de los casos se hizo de forma personalizada, evidenciada en textos con discurso fluido; y 4) es importante mencionar que para facilitar el aprendizaje de la argumentación escrita es imperante privilegiar conceptos y procedimientos de la argumentación, haciendo uso de textos auténticos de una disciplina en particular o producidos por estudiantes, que impliquen reflexión, y que puedan emular las buenas prácticas en géneros textuales y situaciones comunicativas específicas. Por ello la gran relevancia de llevar a cabo las Olimpiadas Escriturales. 


\section{CONCLUSIONES}

De acuerdo al trabajo presentado y a los resultados analizados en el estudio, se pueden plantear las siguientes conclusiones principales:

1.- Los estudiantes asimilaron la estructura textual propuesta, determinada por las diferentes etapas: título, introducción, tesis, argumentos y conclusión.

2.- La retroalimentación efectuada por los mentores durante el proceso de escritura, contribuyó a la apropiación de la estructura textual argumentativa. Además, tener claridad sobre las etapas que la conforman impacta positivamente en las producciones textuales.

3.- La aplicación de este tipo de prácticas de escritura en otras asignaturas, bajo contextos particulares permitiría medir el impacto de la metodología desarrollada.

\section{REFERENCIAS}

Ávila, Y. C., El ensayo académico: algunos apuntes para su estudio, Sapiens: Revista Universitaria de Investigación, 8(1), 147-160 (2007).

Barletta, N. y Chamorro, D., Las unidades textuales: organizadoras del texto, Zona Próxima, (12), 176-189 (2010).

Camps, A y Castelló, M., La escritura académica en la universidad, Revista de Docencia Universitaria, 11(1) 17- 36 (2013).

Carlino, P., Leer textos complejos al comienzo de la educación superior: tres situaciones didácticas para afrontar el dilema. Textos. Didáctica de la Lengua y la Literatura, (33), 43-51 (2003).

Carlino, P., Alfabetización académica diez años después, Revista Mexicana de Investigación educativa, 18(57), 355-381 (2013).

Castells, N., Mateos, M., y otros tres autores, Profiles of self-perceived competencies and conceptions of academic writing in university students/Perfiles de competencias y concepciones sobre la escritura académica en estudiantes universitarios. Cultura y Educación, 27(3), 569-593 (2015).

Díaz, Á., Retórica de la escritura académica: pensamiento crítico y argumentación discursiva. 1ª Ed., 67-159, Universidad de Antioquia. Antioquia, Colombia (2014).

Errázuriz, M., El desarrollo de la escritura argumentativa académica: los marcadores discursivos, doi: 10.7764/onomazein.30.13, Onomázein, 30, 217-236 (2014).

Halliday, M. y Hasan, R., Cohesion in English, 1르 Ed, 230-270, English Language Series, Longman, London (1976).

Halliday, M., Towards a language-based theory learning, Linguistics and Education, 5(2), 93-106 (1993).

Hyland, K., Disciplinary identities: Individuality and community in academic discourse, 1-236, University Press, Cambridge, Reino Unido (2012).

Kruse, O., Perspectives on Academic Writing in European Higher Education: Genres, Practices, and Competences, Revista de Docencia Universitaria, 11, 37-58 (2013).

Lave, J. y Wenger, E. Situated learning. Legitimate peripheral participation, 1를 Ed11-138, Cambridge University Press, New York, USA (1991).

López, C., Benedito, V. y León, M. J., El Enfoque de Competencias en la Formación Universitaria y su Impacto en la Evaluación. La Perspectiva de un Grupo de Profesionales Expertos en Pedagogía, https://doi: 10.4067/S071850062016000400003, Formación Universitaria, 9(4), 11-22 (2016).

Muñoz, C. y Valenzuela, J., Características Psicométricas de una Rúbrica para Evaluar Expresión Escrita a Nivel Universitario, https://doi: 10.4067/S0718-50062015000600010, Formación Universitaria. 8(6), 75-84 (2015)

Padilla, C., y López E., Prácticas de retroalimentación en aulas universitarias de humanidades: Comentarios digitales docentes y perfiles estudiantiles de escritor, doi: 10.4067/S0718-09342019000200330, Revista Signos. Estudios de Lingüística, 52(100), 330-356 (2019).

Páez, A. C., Escritura, discurso y argumentación. Enunciación, 18(1), 94-108 (2013).

Pastene, F. C., Díaz, R. M., y Molina, S. A., Producción del ensayo argumentativo en estudiantes de dos establecimientos de educación media técnico-profesional de la provincia de Nuble: Resultados de un diagnóstico, ISSN: 0716 - 5811, Literatura y lingüística, (34), 395-418 (2016).

Peña, F. J., Leer y escribir. Prácticas necesarias en la universidad, Educere, 15 (52), 711-719 (2011).

Boillos, M.M., La autopercepción de las habilidades escritoras en el inicio de la etapa universitaria, ISSN: 2171-9098, Revista de la Facultad de Educación de Albacete, 33(2), 149-160 (2018).

Ramírez, J. A., y Zamora, J. R., La producción escrita de textos argumentativos en la educación superior, Revista de Lenguas Modernas, (20), 167-181 (2014). 
Rayas, L. T., y Méndez, A. M., Los estudiantes universitarios ante la escritura del ensayo académico: dificultades y posibilidades, Innovación educativa, 17(75), 123-147 (2017).

Raposo, M. y Martínez, E., La rúbrica en la enseñanza universitaria: un recurso para la tutoría de grupos de estudiantes, https://doi: 10.4067/S0718-50062011000400004, Formación universitaria. 4(4),19-28 (2011).

Ruiz, R. F., Textualidad y gramática argumentativa, Cuadernos de Lingüística Hispánica, (24), 25-42 (2014).

Torres, A., ¿Escritura disciplinar? Una experiencia a partir de la lectura crítica y la habilidad argumentativa en la universidad, Revista mexicana de investigación educativa, 23(76), 95-124 (2018).

Van Dijk, T. A., La ciencia del texto. 3ª Ed., 158-163, Paidós Ibérica, Barcelona, España (1992).

Villarroel, C., Garcia-Mila, M., Felton, M., y Miralda-Banda, A., Effect of argumentative goals in the quality of argumentative dialogue and written argumentation, doi: 10.1080/02103702.2018.1550162, Infancia y Aprendizaje, 42(1), 37-86 (2019).

Weston, A., Las claves de la argumentación, 11르 Ed., 19-95, Ariel. Barcelona, España (2006). 
